\title{
Rapid qualitative and quantitative detection of formaldehyde in squids based on colorimetric sensor array
}

\author{
Binbin Guan ${ }^{1, *}$, Hongmei Ding ${ }^{1}$, Bin Chen ${ }^{1}$, Mi Zhou ${ }^{1}$, and Zhaoli Xue ${ }^{2}$ \\ ${ }^{1}$ Nantong Food and Drug Supervision and Inspection Center, Nantong 226001, PR China \\ ${ }^{2}$ School of Chemistry and Chemical Engineering, Jiangsu University, Zhenjiang 212013, PR China
}

\begin{abstract}
The colorimetric sensor array was used to detect the volatile organic compounds (VOCs) in squids with different formaldehyde content. In order to distinguish whether the formaldehyde is artificially added in the squids, the linear discriminant analysis (LDA) and K-nearest neighbor (KNN) based on principal component analysis (PCA) were used to make qualitative judgments, the result shows that the recognition rates of the training set and prediction set of the LDA model were $95 \%$ and $85 \%$ respectively, and the recognition rates of the training set and prediction set of the KNN model were both $90 \%$. Moreover, error back propagation artificial neural network (BP-ANN) was used to quantitatively predict the concentration of formaldehyde in squids. The result indicates that the BP-ANN model acquired a good recognition rate with the correlation coefficient $\left(\mathrm{R}_{\mathrm{p}}\right)$ for prediction was 0.9887 when the PCs was 10 . To verify accuracy and applicability of the model, paired sample t-test was used to verify the difference between the predicted value of formaldehyde in the BP-ANN model and the actual addition amount. Therefore, this approach showed well potentiality to provide a fast, accuracy, no need for a pretreatment, and low-cost technique for detecting the formaldehyde in squids.
\end{abstract}

\section{Introduction}

Squid is more and more popular among consumers because of its unique flavor, rich protein, and various beneficial elements [1-2]. However, in recent years, a large number of studies have reported that squid and its products contain high levels of chemical substances such as formaldehyde, which has caused widespread concern for its food safety [3]. In addition to a small amount of background value contained in squid itself, some illegal vendors have illegally added formaldehyde to squid and its products in order to make profits. Formaldehyde, commonly known as formalin, is a strong carcinogen. Its main function is to preserve the freshness of food and make food white [4]. However, long-term excessive intake of formaldehyde can easily cause cancer and gene mutation [5]. The International Agency for Research on Cancer (IARC) has classified formaldehyde as a carcinogen. So far, many countries in the world have not only regulated the maximum residual amount of formaldehyde in squid, but also developed a variety of conventional detection methods, including spectrophotometry, electrochemistry and chromatography [6-13]. Although these methods have high sensitivity and accurate measurement, they all have the disadvantages of long detection time, high detection cost and complicated pre-processing. These limitations hinder its application in the rapid detection of squid formaldehyde.
In this study, the aim was to develop a rapid and quantitative detection method of formaldehyde content in squid based on electronic nose for screening massive samples and providing a reference for protecting food safety at the stages of aquatic product storage and processing.

\section{Material and Method}

\subsection{Squid samples}

Squid samples were purchased from Metro Supermarket in Nantong, China. Squid samples are divided into six categories, the blank squid sample were separated without water cleaning, marked as S- 0 , and the positive squid samples were stochastically soaked in different concentrations of formaldehyde solution $(1 \%, 2 \%, 4 \%$, $6 \%, 8 \%$, which marked as S-1, S-2, S-3, S-4 and S-5 respectively) for 12 hours, 10 samples of each type, with a total of 60 samples.

\subsection{Colorimetric sensor array}

The colorimetric sensor array consists of five porphyrins and four $\mathrm{pH}$ indicators. Among them, porphyrins were synthesized in our laboratory, including: 5, 10, 15, 20Tetraphenyl-21H, 23H-porphine zinc, 5, 10, 15, 20Tetraphenyl-21H, 23H-porphine manganese, $5,10,15$, 20-Tetraphenyl-21H, 23H-porphine copper, 5, 10, 15,

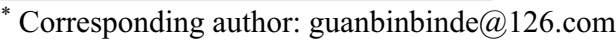


20-Tetraphenyl-21H, 23H-porphine nickel and 5, 10, 15, 20-Tetraphenyl-21H, 23H-porphine cobalt. The four $\mathrm{pH}$ indicators (Neutral red, Phenol blue, Bromocresol Green and Methyl red) were obtained from Sinopharm Chemical Reagent Co., Ltd.

The colorimetric sensor array was made as follows: (1) porphyrins were dissolved in trichloromethane, and the $\mathrm{pH}$ indicators were dissolved in ethanol, the concentration is $1 \mathrm{mg} / \mathrm{mL}$; (2) after ultrasound for one hour, colorimetric dye solutions were obtained; (3) 9 colorimetric dyes were printed on the hydrophobic polyvinylidene fluoride (PVDF) membrane (Merck Millipore, Germany) using micro-capillary pipettes, constructing a $3 \times 3$ sensor array; (4) after 15 min drying in the fume hood, the colorimetric sensor array could be obtained and maintained in a closed environment for further process.

\subsection{Artificial olfaction system}

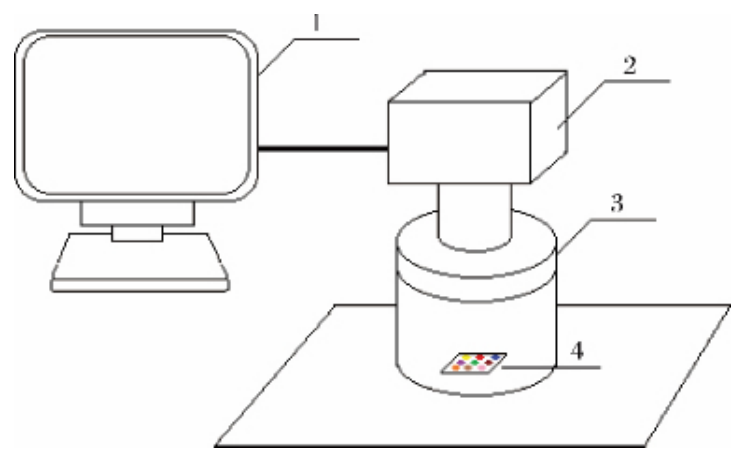

Fig.1 Artificial olfaction system (1-computer, 2-camera, 3light source, 4-colorimetric sensor array)

Fig.1 is a schematic diagram of a self-made artificial olfaction system. The device uses a ring light source to ensure the uniform image brightness, the camera acquired the image of the sensor array before it was exposed to the VOCs, and recorded it as the before image. Then, put the squid sample in the fresh-keeping box, fix the sensor array on the fresh-keeping box lid, and close the lid and start timing. With the sensor array exposed to the VOCs of squid samples for ten minutes, color changes occurred in some colorimetric dyes and the color change of colorimetric sensor was recorded with the camera as the after image. Response difference of before and after image is processed by a specific data processing software.

\subsection{Multivariate statistical analysis}

Multivariate analysis methods play a key role in characterizing formaldehyde in squid samples based on the colorimetric sensor array. The PCA, LDA, KNN, and PLS algorithms were implemented in Matlab R2010a, the BP-ANN algorithm was implemented in NeuroShell 2 , the paired sample t-test was implemented in SPSS Statistics 17.

\section{Results and discussion}

\subsection{Characterization of squid samples based on colorimetric sensor array}

The detection result of the colorimetric sensor array is the color change of the color-sensitive sensor before and after the reaction. This technology converts olfactory information into visual information, so that the smell is "visible". Compared with traditional method, olfactory imaging is more intuitive and vivid. Fig. 2 shows the difference maps before and after the reaction of squid samples which soaked in different formaldehyde concentrations. As we can see from the figure, there is a significant difference between the difference map of squid samples that not soaked in formaldehyde (S-0) and squid samples that soaked in formaldehyde (S-1, S-2, S$3, \mathrm{~S}-4, \mathrm{~S}-5)$. In addition, the color of No. 5 sensor in the response difference maps from S-1 to $\mathrm{S}-5$ is getting brighter, which corresponds to the formaldehyde concentration.
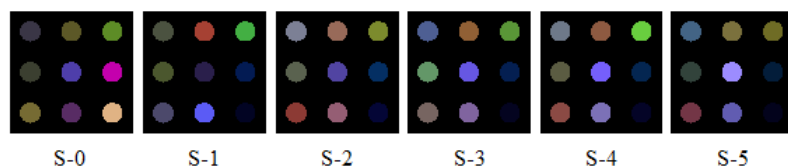

Fig.2 Characteristic images for the squids soaked in different concentrations of formaldehyde solution

\subsection{Principal component analysis (PCA)}

Principal components analysis (PCA) is a mathematical transformation utilized to extract the variance among entries in a data matrix by reducing the redundancy in the dimensionality of the data, which could transform a group of potentially correlated variables into a group of linearly uncorrelated variables through orthogonal transformation [14]. This group of variables after conversion is called principal components. PCA is more like a preprocessing method, which can simplify data well. It takes the data points (changes in RGB values for each colorimetric sensor in the array) from all the squid samples and generates a set of orthogonal eigenvectors (principal components, PCs) for maximum variance.

Fig.3(a) shows a 3-dimensional (3D) space of all the squids samples represented by PC1 (the first principal component), $\mathrm{PC} 2$ (the second principal component) and PC3 (the third principal component). PC1 contains $91.78 \%$ of the variance of the data, PC2 contains $4.55 \%$ and the first three components represent $98.00 \%$ of the total variance. Geometrical exploration based on PCA score plots shows the clusters' trend in the 3-dimension space. From the picture, the squids that has not been soaked in formaldehyde can be clearly distinguished from the squids that has been soaked in formaldehyde, at the same time, the squid samples immersed in different concentrations of formaldehyde also have a certain aggregation trend in the figure. 
(a)
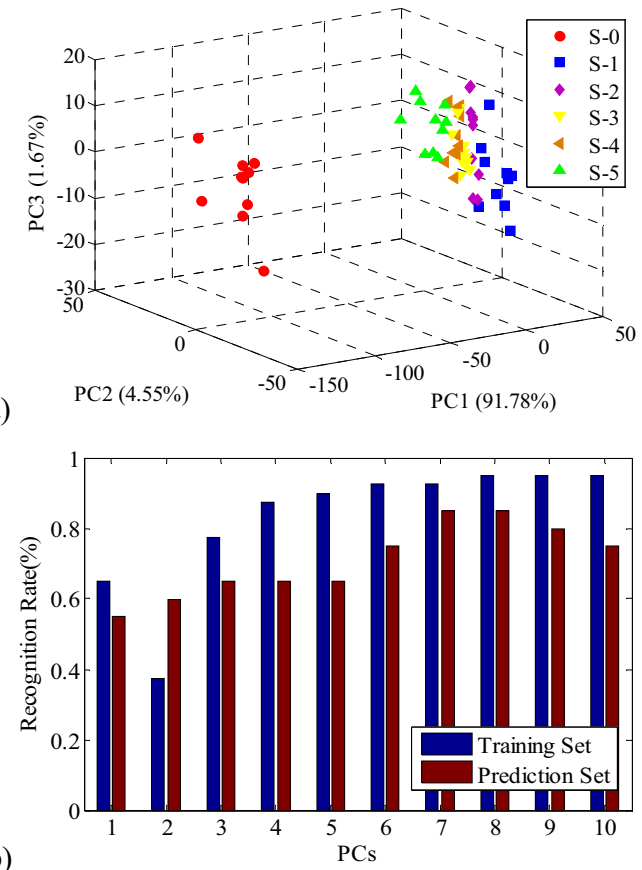

(b)

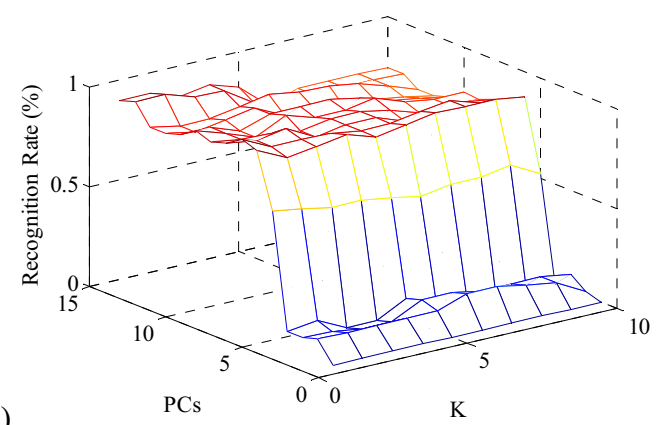

(c)

Fig.3 (a) Classification result achieved by PCA (b) Discriminant results of LDA under different principal component numbers (c) Discriminant results of KNN model under different principal component numbers and $\mathrm{K}$ values

\subsection{Qualitative analysis of colorimetric sensor array for formaldehyde detection in squids}

\subsubsection{Linear discriminant analysis (LDA)}

Linear discriminant analysis (LDA) is one of the simplest and most commonly used classification methods. Its goal is to search for the mapping line that can best classify the two types of samples, and select the linear combination representing the original variables in a way that maximizes the ratio of the dispersion between the two classes after the mapping, and finally realize the clustering of the samples within the class and the relative dispersion of the samples between the classes [15].

We choose two-thirds of all the samples, namely 40 samples, as the training set and one-third of the samples as the prediction set to establish a squid LDA classification discriminant model. As we can see from Fig.3(b), if all the squid samples are divided into 6 categories according to the added formaldehyde concentration, the model has the best recognition rate when the PCs is 8 . At this time, the recognition rate of the training set is $95 \%$, and the recognition rate of the prediction set is $85 \%$. The result shows that the selected characteristic variables can be effectively used to distinguish squid which containing different concentrations of formaldehyde.

\subsubsection{K-nearest neighbor (KNN)}

K-nearest neighbor (KNN) is a classic pattern recognition method based on the similarity of samples in the pattern space. The basic principle is to calculate the similarity of each sample in the prediction set and the correction set in a multi-dimensional space, and find out $\mathrm{K}$ in the prediction set according to the similarity [16]. The nearest neighbor correction set samples, and then the $\mathrm{K}$ nearest neighbor samples are judged according to the principle of minority obeys the majority, and then the attribution situation of the prediction set samples is obtained. In this research, we choose two-thirds of the samples of each group as the training set and one-third of the samples as the prediction set to establish a squid KNN classification discriminant model.

The result in Fig.3(c) shows that when the best $\mathrm{K}$ value is 1 and the best principal component is 15 , the recognition rate of training set and prediction set are both $90 \%$. The result indicate that the model has a good recognition rate, and this method could be used for the classification of whether the squid is soaked in formaldehyde.

\subsection{Quantitative analysis of colorimetric sensor array for formaldehyde detection in squids}

Table 1. BP-ANN model results of formaldehyde concentration with different PCs

\begin{tabular}{ccc}
\hline \multirow{2}{*}{ PCs } & \multicolumn{2}{c}{ correlation coefficient } \\
\cline { 2 - 3 } & $R_{c}$ & $R_{p}$ \\
4 & 0.9984 & 0.9880 \\
5 & 1 & 0.9887 \\
6 & 1 & 0.9887 \\
7 & 0.9998 & 0.9868 \\
8 & 0.9999 & 0.9882 \\
9 & 0.9903 & 0.7633 \\
10 & 0.9996 & 0.9867 \\
\hline
\end{tabular}

The back propagation artificial neural network (BP-ANN) model is established with the principal component after PCA analysis as the model input and the actual formaldehyde soak concentrations as the network output [17]. With a random sample of two-thirds of all the 60 samples as the training set and the remaining 20 samples for the prediction set data. The number of training iterations is set to 100; the transfer function from the input layer to the hidden layer and from the hidden layer to the output layer is tanh function; the network learning rate is 0.1 ; the momentum factor is 0.1 , and the initial weight value of 0.3 . 
Table 1 is the BP-ANN results of formaldehyde concentration under 4-10 PCs. It can be seen from Table 1 that when the PCs is 5, the correlation coefficient of formaldehyde in the training set and the prediction set are the highest compared with the actual added amount, and the model is the best, at this time, Rc of the training set is 1 , the RMSECV value is $2.9992 \times 10^{-5}$, and the $\mathrm{Rp}$ of the prediction set is 0.99887 and the RMSEP value is $4.5883 \times 10^{-3}$.

\subsection{The accuracy test of formaldehyde quantitative model paired sample t-test}

To verify accuracy and applicability of the model, paired sample t-test was used to verify the pairwise difference between the predicted value of formaldehyde in the BPANN model and the actual addition amount. As we can see from Table 2, Sig. value of the training set and prediction set were both bigger than 0.05 , hence, there was no significant difference between predicted values and actual values.

Table 2. The results of paired sample t-test

\begin{tabular}{|c|c|c|c|}
\hline \multicolumn{2}{|c|}{ Samples } & Training set & Prediction set \\
\hline \multirow{6}{*}{$\begin{array}{l}\text { Pairwise } \\
\text { difference }\end{array}$} & Mean & 0.0000 & 0.0010 \\
\hline & $\begin{array}{l}\text { Standard } \\
\text { deviation }\end{array}$ & 0.0003 & 0.0047 \\
\hline & $\begin{array}{l}\text { Standard } \\
\text { error of the } \\
\text { mean }\end{array}$ & 0.0000 & 0.0010 \\
\hline & $\mathrm{t}$ & 0.000 & 0.998 \\
\hline & df & 39 & 19 \\
\hline & Sig. & 1.000 & 0.331 \\
\hline
\end{tabular}

\section{Conclusion}

A colorimetric sensor array was designed for the detection the concentration of formaldehyde in squid samples. The array is composed of five porphyrins materials and four $\mathrm{pH}$ indicators immobilized on PVDF. Response data of before and after image were analyzed with PCA analysis, the result indicate that the squids that has not been soaked in formaldehyde can be clearly distinguished from the squids which has been soaked in formaldehyde. LDA and KNN model showed good characterization skills when they were used to distinguish whether the formaldehyde had been artificially added in squids. What's more, BP-ANN was used to quantitatively predict the concentration of formaldehyde in squids, and paired sample t-test was used to verify the difference between the predicted value and the actual addition amount, the result shows the Rp was 0.9887 and there was no significant difference between predicted values and actual values. Therefore, the colorimetric sensor array could be well used in rapid qualitative and quantitative detection of formaldehyde in squids.

\section{Funding Information}

This work has been financially supported by Foundation for the Author of Jiangsu Natural Science Foundation of P.R. China (BK20190238), Nantong Science and Technology Foundation (MS12017018-1), JiangSu Food and Drug Administration Project (20180224).

\section{References}

1. S.S. Wu, J.L. Zhu, P. Shen, et al., J. Chin. Inst. Food Sci. Technol. 5,148-156 (2014)

2. A.I. Arkhipkin, P.G.K. Rodhouse, G.J. Pierce, et al., Rev. Fish. Sci. Aquac. 23(2),92-252 (2017)

3. A.S. Aminah, H. Zailina, A.B. Fatimah. Food Publ. Health 3(1), 52-58 (2013)

4. N. Noordiana, A.B. Fatimah, Y.C.B Farhana. Int. Food Res. J. 18(1),125-136 (2011)

5. H.P. Gelbke, H. Buist, R. Eiset, et al., Food Chem. Toxicol. 132,110598 (2019)

6. J.J Ma, D.Q. Zhou, S.F Liu, et al., Marine Fisheries Research 26(1),28-31 (2005)

7. T.S. Yeh, T.C. Lin, C.C. Chen, et al., J. Food Drug Anal. 21(2),190-197 (2013)

8. M.D.M. Abadi, N. Ashraf, M. Chamsaz, et al., Talanta 99,1-12 (2012)

9. S.N. Azizi, S. Ghasemi, F. Amiripour. Sens. Actuators B 227,1-10 (2016)

10. SC/T 3025-2006 Determination of formaldehyde in aquatic products (2006)

11. P. Wahed, M.A. Razzaq, S. Dharmapuri, et al., Food Chem. 202,476-483 (2016)

12. D.C. Gu, W. Liu, Y. Yan, et al., LWT 101,382-388 (2019)

13. W. Wongniramaikul, W. Limsakul, A. Choodum, et al., Food Chem. 249,154-161 (2018)

14. A.H. Sahoolizadeh, B.Z. Heidari, C.H. Dehghani. Int. J. Comput. Sci. Eng. 2(4),218-223 (2008)

15. L.F. Chen, H.Y.M. Liao, M.T. Ko, et al. Pattern Recognit. 33(10), 1713-1726 (2000)

16. C.X. Wang, Z.M. Pan, C.S. Ma, et al., Comput. Eng. 38(20), 160-163 (2012)

17. K. Yetimezsoy, S. Demirel, J. Hazard. Mater. 153(3),1288-1300 (2008) 